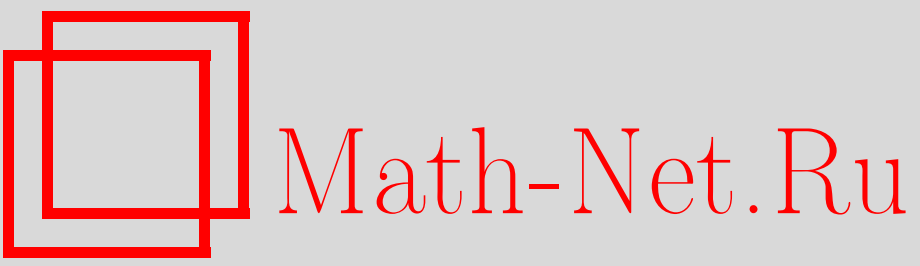

М. Е. Палистрант, Влияние магнитной примеси на сверхпроводимость в системах со сравнимыми значениями энергии Ферми и энергии Дебая, ТМФ, 1999, том 119, номер 3, 455-474

DOI: https://doi.org/10.4213/tmf752

Использование Общероссийского математического портала Math-Net.Ru подразумевает, что вы прочитали и согласны с пользовательским соглашением

http: //www . mathnet.ru/rus/agreement

Параметры загрузки:

IP : 54.198 .187 .58

26 апреля 2023 г., 17:53:24 


\section{ВЛИЯНИЕ МАГНИТНОЙ ПРИМЕСИ НА СВЕРХПРОВОДИМОСТЬ В СИСТЕМАХ СО СРАВНИМЫМИ ЗНАЧЕНИЯМИ ЭНЕРГИИ ФЕРМИ И ЭНЕРГИИ ДЕБАЯ}

Получена основная система уравнений теории сверхпроводимости для систем с хаотически распределенной парамагнитной примесью замещения, в которых нарушается теорема Мигдала (нельзя считать $\omega_{\mathrm{D}} \ll E_{\mathrm{F}}$ ). Учтены электрон-фононные и примесные диаграммы, а также дополнительные диаграммы, соответствующие пересечению электрон-фононных и электрон-примесных линий. В предельном случае слабой электрон-ффононной связи получено уравнение для температуры сверхпроводящего перехода $T_{C}$, которое отличается от случая обычных сверхпроводников перенормировкой величины $T_{C 0}$ и параметра примесного рассеяния $\rho$. Эти величины существенно зависят от параметра Мигдала $m=\omega_{\mathrm{D}} / E_{\mathrm{F}}$ и передаваемого импульса $q_{c}$. Показано, что убывание величины $T_{C}$ с ростом концентрации примеси замедляется по сравнению со случаем обычных сверхпроводников и степень этого замедления определяется величинами $m$ и $q_{c}$. Вычислен также изотопический коэффициент $\alpha$, поведение которого как функции концентрации примеси зависит от величин $m$ и $q_{c}$.

\section{1. ВВЕДЕНИЕ}

Характерной особенностью высокотемпературных керамик, соединений $\mathrm{A}_{3} \mathrm{C}_{60}$, а также органических сверхпроводников являются малые значения энергии Ферми $E_{\mathrm{F}}$. Эти значения могут быть порядка $0.1-0.3$ эВ $[1,2]$. При этом частота Дебая $\omega_{\mathrm{D}} \approx 0.08-$ 0.16 эВ [3]. Таким образом, нарушается теорема Мигдала [4], поскольку нельзя считать пренебрежимо малым отношение $\omega_{\mathrm{D}} / E_{\mathrm{F}}$, т.к. энергии электронов и фононов сравнимы. Это обстоятельство приводит к необходимости обобщить теорию сверхпроводимости Бардина-Купера-Шриффера-Боголюбова с учетом дополнительных многочастичных эффектов. Такие исследования выполнены в работах $[3,5]$, где получено уравнение Элиашберга [6] с включением первого порядка по параметру неадиабатичности $\lambda \omega_{\mathrm{D}} / E_{\mathrm{F}}$, т.е. с учетом вершинных и пересекаюшихся диаграмм электрон-фононного

* Институт прикладной физики АН Республики Молдова, Кишинев, Республика Молдова 
взаимодействия. Было показано, что происходят существенная перенормировка константы электрон-фононного взаимодействия в уравнениях Элиашберга и увеличение температуры сверхпроводящего перехода при малых значениях импульса обрезания $q_{c}$ электрон-фононного взаимодействия. При $\lambda \approx 0.5-1$ с учетом эффектов неадиабатичности величина $T_{C}$ достигает значений, соответствуюших $\lambda \approx 3$ в уравнении Элиашберга с сильной электрон-фононной связью. Область малых значений величины $q_{c}$ определяется специфическими свойствами материалов и, в частности, наличием сильных корреляций электронов в системе.

Важной проблемой в современных исследованиях материалов высокотемпературной сверхпроводимости (ВТСП) является изучение влияния примеси на сверхпроводяшие свойства оксидных керамик.

В случае изотропной модели величина $T_{C}$ и другие термодинамические величины не зависят от концентрации немагнитной примеси (теорема Андерсона [7]). Магнитная же примесь подавляет сверхпроводимость в соответствии с теорией Абрикосова и Горькова [8], которая основывается на теории сверхпроводимости Бардина-Купера-Шриффеpa. Теория [8] применяется в настоящее время для описания влияния магнитной примеси на сверхпроводимость в ВТСП-материалах. Возникает вопрос о том, насколько это корректно и как изменяется примесная зависимость величины $T_{C}$, если учесть эффекты неадиабатичности, о которых шла речь вьше. Решению этой проблемы посвяшена данная работа. Мы рассматриваем совместное действие магнитной примеси и электрон-фононного взаимодействия в системе. Наряду с пересекаюшимися диаграммами электрон-фононного взаимодействия $[3,5]$ мы учитываем также примесные диаграммы и диаграммы с пересечением электрон-фононных и примесных линий.

Работа построена следующим образом. Во втором разделе приведен гамильтониан системы и вводятся функции Грина (нормальные и аномальные). Основываясь на теории возмушений и выполнив усреднение по положениям хаотически распределенной примеси и ориентациям спинов, мы получаем систему уравнений для функций Грина и выражения для массовых операторов. В разделе 3 рассмотрен случай $T \approx T_{C}$, что позволяет ограничиться диаграммами, линейными по параметру порядка. Выполнено усреднение по поверхности Ферми и приведены основные уравнения сверхпроводимости с учетом перенормировки константы электрон-фононного и примесного взаимодействий. В разделе 4 рассмотрен предел слабой связи, дана оценка вклада неадиабатического эффекта (дополнительных диаграмм) и получено уравнение для температуры сверхпроводящего перехода. Рассмотрен предел малых и больших концентраций примеси. Получены также выражения для критической концентрации примеси и изотопического эффекта. В заключении сформулированы результаты исследования.

\section{2. ГАМИЛЬТОНИАН И УРАВНЕНИЯ ДЛЯ ФУНКЦИЙ ГРИНА}

Мы рассматриваем систему с парамагнитной примесью, описываемую гамильтониаHOM

$$
H=H_{0}+H_{1}+H_{2},
$$


где $H_{0}$ - гамильтониан свободных электронов и фононов, а

$$
\begin{aligned}
& H_{1}=\sum_{\sigma} \int d \vec{x} \psi_{\sigma}^{+}(\vec{x}) \psi_{\sigma}(\vec{x}) \varphi(\vec{x}), \\
& H_{2}=\sum_{\alpha, \beta} \int d \vec{x} \psi_{\alpha}^{+}(\vec{x}) V_{\alpha \beta}(\vec{x}) \psi_{\beta}(\vec{x})
\end{aligned}
$$

- соответственно гамильтонианы электрон-фононного и электрон-примесного взаимодействий (здесь $\psi_{\alpha}(\vec{x})$ - оператор уничтожения электрона спина $\alpha$ в положении $\vec{x}, \varphi(\vec{x})-$ фононный оператор). Взаимодействие электрона с $n$-м парамагнитным примесным атомом с классическим спином $\vec{S}$ в точке $\vec{R}_{n}$ описьвается потенциалом [8]

$$
V_{\alpha \beta}(\vec{x})=\sum_{n} V_{\alpha \beta}\left(\vec{x}-\vec{R}_{n}\right)=V_{1}(\vec{x}) \delta_{\alpha \beta}+\frac{1}{2} \vec{S} \vec{\sigma}_{\alpha \beta} V_{2}(\vec{x})
$$

Здесь $\vec{\sigma}$ - спин-матричный вектор, а $V_{1}$ и $V_{2}$ - потенциалы рассеяния электронов на примеси (немагнитной и магнитной, соответственно).

Введем температурные (мацубаровские) одноэлектронные функции Грина

$$
\begin{aligned}
G_{\beta \alpha}\left(x x^{\prime}\right) & =-\left\langle T \psi_{\beta}(x) \psi_{\alpha}^{+}\left(x^{\prime}\right)\right\rangle, \\
\widetilde{F}_{\alpha \alpha^{\prime}}\left(x x^{\prime}\right) & =-\left\langle T \psi_{\alpha}^{+}(x) \psi_{\alpha^{\prime}}^{+}\left(x^{\prime}\right)\right\rangle, \\
F_{\beta \beta^{\prime}}\left(x x^{\prime}\right) & =-\left\langle T \psi_{\beta}(x) \psi_{\beta^{\prime}}\left(x^{\prime}\right)\right\rangle,
\end{aligned}
$$

а также фононную функцию Грина

$$
D\left(x x^{\prime}\right)=-\left\langle T_{\varphi}(x) \varphi\left(x^{\prime}\right)\right\rangle
$$

Здесь $x=(\vec{x}, \tau)$, а $\psi_{\alpha}(x)=e^{\tau H} \psi_{\alpha}(\vec{x}) e^{-\tau H}, \psi_{\alpha}^{+}(x)=e^{\tau H} \psi_{\alpha}^{+}(\vec{x}) e^{-\tau H}-$ гайзенберговские операторы частиц; $\langle\ldots\rangle$ означает усреднение по состоянию системы взаимодействующих частиц.

Исследуем совместное влияние электрон-фононного и примесного взаимодействий на функции Грина (4). С этой целью перейдем к представлению взаимодействия и применим теорию возмушений [9]. Рассматривая члены $H_{1}+H_{2}$ как возмушение, запишем ряд теории возмушений для функций (4). Выполним затем усреднение по положениям $\vec{R}_{n}$ хаотически распределенной магнитной примеси и ориентациям спинов $\vec{S}$, воспользовавшись соотношением

$$
\begin{aligned}
\overline{V_{\alpha_{1} \beta_{1}}\left(\vec{x}_{1}\right) V_{\alpha_{2} \beta_{2}\left(\vec{x}_{2}\right)}=} & \frac{c}{V} \sum_{\vec{q}} e^{-i \vec{q}\left(\vec{x}_{1}-\vec{x}_{2}\right)} \times \\
& \times\left[\left|V_{1}(\vec{q})\right|^{2}+\frac{1}{12} S(S+1)\left|V_{2}(\vec{q})\right|^{2} \sum_{i} \sigma_{\alpha_{1} \beta_{1}}^{i} \sigma_{\alpha_{2} \beta_{2}}^{i}\right] .
\end{aligned}
$$


Подставим (6) в указанные выше ряды теории возмушений для функций Грина (4) и выполним суммирование по спиновым переменным. При этом используем для функций Грина в случае отсутствия спиновых взаимодействий (без примеси) соотношения

$$
\begin{aligned}
& G_{\beta \alpha}\left(x x^{\prime}\right)=G\left(x x^{\prime}\right) \delta_{\beta \alpha}, \\
& \widetilde{F}_{\alpha \alpha^{\prime}}\left(x x^{\prime}\right)=-g_{\alpha \alpha^{\prime}} \widetilde{F}\left(x x^{\prime}\right), \\
& F_{\alpha \alpha^{\prime}}\left(x x^{\prime}\right)=g_{\alpha \alpha^{\prime}} F\left(x x^{\prime}\right),
\end{aligned}
$$

где

$$
g_{\alpha \alpha^{\prime}}=\delta_{\alpha,-\alpha^{\prime}}\left(\delta_{\alpha \uparrow}-\delta_{\alpha \downarrow}\right)=i\left(\tau_{2}\right)_{\alpha \alpha^{\prime}} .
$$

Таким образом, в результате довольно громоздких вычислений получаем систему уравнений для полных функций Грина $\bar{G}, \overline{\widetilde{F}}$ и $\bar{F}$, усредненных по положениям атомов примеси и ориентациям их спинов и учитывающих неадиабатический эффект и наличие парамагнитной примеси. Эти уравнения имеют вид

$$
\begin{aligned}
\bar{G}\left(x, x^{\prime}\right)= & G\left(x, x^{\prime}\right)+\iint d x_{1} d x_{2} \times \\
& \times\left[G\left(x, x_{1}\right) M_{i}\left(x_{1}, x_{2}\right) \bar{G}\left(x_{2}, x^{\prime}\right)-G\left(x, x_{1}\right) \Sigma\left(x_{1}, x_{2}\right) \overline{\widetilde{F}}\left(x_{2}, x^{\prime}\right)-\right. \\
& \left.-F\left(x, x_{1}\right) \widetilde{\Sigma}\left(x_{1}, x_{2}\right) \bar{G}\left(x_{2}, x^{\prime}\right)-F\left(x, x_{1}\right) M_{i}^{\prime}\left(x_{2}, x_{1}\right) \overline{\widetilde{F}}\left(x_{2}, x^{\prime}\right)\right], \\
\overline{\widetilde{F}}\left(x, x^{\prime}\right)= & \widetilde{F}\left(x, x^{\prime}\right)+\iint d x_{1} d x_{2} \times \\
& \times\left[G\left(x_{1}, x\right) \widetilde{\Sigma}\left(x_{1}, x_{2}\right) \bar{G}\left(x_{2}, x^{\prime}\right)+G\left(x_{1}, x\right) M_{i}^{\prime}\left(x_{2}, x_{1}\right) \overline{\widetilde{F}}\left(x_{2}, x^{\prime}\right)+\right. \\
& \left.+\widetilde{F}\left(x, x_{1}\right) M_{i}\left(x_{1}, x_{2}\right) \bar{G}\left(x_{2}, x^{\prime}\right)-F\left(x, x_{1}\right) \Sigma\left(x_{1}, x_{2}\right) \overline{\widetilde{F}}\left(x_{2}, x^{\prime}\right)\right], \\
\bar{F}\left(x, x^{\prime}\right)= & F\left(x, x^{\prime}\right)+\iint d x_{1} d x_{2} \times \\
& \times\left[G\left(x, x_{1}\right) M_{i}\left(x_{1}, x_{2}\right) \bar{F}\left(x_{2}, x^{\prime}\right)+F\left(x, x_{1}\right) M_{i}^{\prime}\left(x_{2}, x_{1}\right) \bar{G}\left(x^{\prime}, x_{2}\right)+\right. \\
& \left.+G\left(x, x_{1}\right) \Sigma\left(x_{1}, x_{2}\right) \bar{G}\left(x^{\prime}, x_{2}\right)-F\left(x, x_{1}\right) \widetilde{\Sigma}\left(x_{1}, x_{2}\right) F\left(x_{2}, x^{\prime}\right)\right] .
\end{aligned}
$$

Здесь $M_{i}, M_{i}^{\prime}, \Sigma$ и $\widetilde{\Sigma}$ - массовые операторы, возникающие из-за взаимодействия с примесью и содержащие наряду с обычными примесными диаграммами также и диаграммы, соответствуюшие пересечению линий примесного и электрон-фононного взаимодействий, $x=(\vec{x}, \tau), d x=d \vec{x} d \tau$.

$\mathrm{B}(\vec{k}, \Omega)$-представлении уравнения (8)-(10) приобретают вид

$$
\begin{aligned}
\bar{G}(k)= & G(k)+G(k)\left[M_{i}(k) \bar{G}(k)-\Sigma(k) \overline{\widetilde{F}}(k)\right]- \\
& -\widetilde{F}(k)\left[\widetilde{\Sigma}(k) \bar{G}(k)+M_{i}^{\prime}(-k) \overline{\widetilde{F}}(k)\right], \\
\overline{\widetilde{F}}(k)= & \widetilde{F}(k)+\overline{\widetilde{F}}(k)\left[M_{i}(k) \bar{G}(k)-\Sigma(k) \overline{\widetilde{F}}(k)\right]+ \\
& +G(-k)\left[M_{i}^{\prime}(-k) \overline{\widetilde{F}}(k)+\widetilde{\Sigma}(k) \bar{G}(k)\right], \\
\bar{F}(k)= & F(k)+G(k)\left[M_{i}(k) \bar{F}(k)+\Sigma(k) \bar{G}(-k)\right]+ \\
& +F(k)\left[M_{i}^{\prime}(-k) \bar{G}(-k)-\Sigma(k) \bar{F}(k)\right],
\end{aligned}
$$


где $k=(\vec{k}, \Omega)$. Функции $G, \widetilde{F}$ и $F$, входящие в (8)-(11), являются функциями Грина чистого сверхпроводника с учетом неадиабатичных эффектов и в $(\vec{k}, \Omega)$-представлении имеют вид

$$
G(k)=-\frac{i \Omega+\varepsilon_{k}+M_{0}(-k)}{A_{0}(k)}, \quad \widetilde{F}(k)=\frac{\widetilde{\Sigma}_{0}(k)}{A_{0}(k)}, \quad F(k)=\frac{\Sigma_{0}(k)}{A_{0}(k)},
$$

где

$$
A_{0}(k)=\left[i \Omega-\varepsilon_{k}-M_{0}(k)\right]\left[-i \Omega-\varepsilon_{k}-M_{0}(-k)\right]+\Sigma_{0}(k) \widetilde{\Sigma}_{0}(k) .
$$

Здесь $M_{0}, \widetilde{\Sigma}_{0}$ и $\Sigma_{0}$ - массовые операторы чистого сверхпроводника с учетом вершинных поправок и пересекаюшихся диаграмм (неадиабатических эффектов) [5]. Решение системы (11) с учетом определений (12) дает

$$
\bar{G}(k)=-\frac{i \Omega+\varepsilon_{k}+M(-k)}{A(k)}, \quad \overline{\widetilde{F}}(k)=\frac{\widetilde{\Xi}(k)}{A(k)}, \quad \bar{F}(k)=\frac{\Xi(k)}{A(k)},
$$

где

$$
\begin{gathered}
M(k)=M_{0}(k)+M_{i}(k), \quad \widetilde{\Xi}(k)=\widetilde{\Sigma}_{0}(k)+\widetilde{\Sigma}(k), \quad \Xi(k)=\Sigma_{0}(k)+\Sigma(k), \\
A(k)=\left|i \Omega+\varepsilon_{k}+M(-k)\right|^{2}+|\Xi(k)|^{2} .
\end{gathered}
$$

Операторы $M(k), \widetilde{\Xi}(k)$ и $\Xi(k)$ являются полными массовыми операторами и включают в себя как диаграммы с электрон-фононным взаимодействием, так и примесные (в том числе и с пересечением линий примесного и электрон-фононного взаимодействий).

Массовые операторы $M(k)$ и $\Xi(k)$ имеют вид

$$
\begin{aligned}
M(\vec{p}, \Omega)= & -\frac{1}{\beta V} \sum_{\vec{p}_{1}, \Omega_{1}} D\left(\vec{p}-\vec{p}_{1}, \Omega-\Omega_{1}\right) \bar{G}\left(\vec{p}_{1}, \Omega_{1}\right)+ \\
& +\frac{1}{(\beta V)^{2}} \sum_{\vec{p}_{1}, \Omega_{1}} \sum_{\vec{p}_{2}, \Omega_{2}} D\left(\vec{p}-\vec{p}_{1}, \Omega-\Omega_{1}\right) D\left(\vec{p}-\vec{p}_{2}, \Omega-\Omega_{2}\right) \times \\
& \times\left[\bar{G}\left(\vec{p}_{1}, \Omega_{1}\right) \bar{G}\left(\vec{p}_{1}+\vec{p}_{2}-\vec{p}, \Omega_{1}+\Omega_{2}-\Omega\right) \bar{G}\left(\vec{p}_{2}, \Omega_{2}\right)-\right. \\
& -\bar{F}\left(\vec{p}_{1}, \Omega_{1}\right) \overline{\widetilde{F}}\left(\vec{p}_{1}+\vec{p}_{2}-\vec{p}, \Omega_{1}+\Omega_{2}-\Omega\right) \bar{G}\left(\vec{p}_{2}, \Omega_{2}\right)- \\
& -\bar{G}\left(\vec{p}_{1}, \Omega_{1}\right) \bar{F}\left(\vec{p}_{1}+\vec{p}_{2}-\vec{p}, \Omega_{1}+\Omega_{2}-\Omega\right) \overline{\widetilde{F}}\left(\vec{p}_{2}, \Omega_{2}\right)- \\
& \left.-\bar{F}\left(\vec{p}_{1}, \Omega_{1}\right) \bar{G}\left(\vec{p}-\vec{p}_{1}-\vec{p}_{2}, \Omega-\Omega_{1}-\Omega_{2}\right) \overline{\widetilde{F}}\left(\vec{p}_{2}, \Omega_{2}\right)\right]+ \\
& +\frac{1}{\beta V^{2}} \sum_{\vec{p}_{1}, \Omega_{1}} \sum_{\vec{p}_{2}} D\left(\vec{p}-\vec{p}_{1}, \Omega-\Omega_{1}\right) \times \\
& \times\left[-W_{+}\left(\vec{p}-\vec{p}_{2}\right) \bar{G}\left(\vec{p}_{1}, \Omega_{1}\right) \bar{G}\left(\vec{p}_{1}+\vec{p}_{2}-\vec{p}, \Omega_{1}\right) \bar{G}\left(\vec{p}_{2}, \Omega\right)+\right. \\
& +W_{-}\left(\vec{p}-\vec{p}_{2}\right) \bar{F}\left(\vec{p}_{1}, \Omega_{1}\right) \bar{G}\left(\vec{p}-\vec{p}_{1}-\vec{p}_{2},-\Omega_{1}\right) \overline{\widetilde{F}}\left(\vec{p}_{2}, \Omega\right)+ \\
& +W_{+}\left(\vec{p}-\vec{p}_{2}\right) \bar{G}\left(\vec{p}_{1}, \Omega_{1}\right) \bar{F}\left(\vec{p}_{1}+\vec{p}_{2}-\vec{p}, \Omega_{1}\right) \overline{\widetilde{F}}\left(\vec{p}_{2}, \Omega\right)+
\end{aligned}
$$




$$
\begin{aligned}
& \left.+W_{-}\left(\vec{p}-\vec{p}_{2}\right) \bar{F}\left(\vec{p}_{1}, \Omega_{1}\right) \overline{\widetilde{F}}\left(\vec{p}_{1}+\vec{p}_{2}-\vec{p}, \Omega_{1}\right) \bar{G}\left(\vec{p}_{2}, \Omega\right)\right]+ \\
& +\frac{1}{\beta V^{2}} \sum_{\vec{p}_{2}, \Omega_{2}} \sum_{\vec{p}_{1}} D\left(\vec{p}-\vec{p}_{2}, \Omega-\Omega_{2}\right) \times \\
& \times\left[-W_{+}\left(\vec{p}-\vec{p}_{1}\right) \bar{G}\left(\vec{p}_{1}, \Omega\right) \bar{G}\left(\vec{p}_{1}+\vec{p}_{2}-\vec{p}, \Omega_{2}\right) \bar{G}\left(\vec{p}_{2}, \Omega_{2}\right)+\right. \\
& +W_{+}\left(\vec{p}-\vec{p}_{1}\right) \bar{F}\left(\vec{p}_{1}, \Omega\right) \overline{\widetilde{F}}\left(\vec{p}_{1}+\vec{p}_{2}-\vec{p}, \Omega_{2}\right) \bar{G}\left(\vec{p}_{2}, \Omega_{2}\right)+ \\
& +W_{-}\left(\vec{p}-\vec{p}_{1}\right) \bar{G}\left(\vec{p}_{1}, \Omega\right) \bar{F}\left(\vec{p}_{1}+\vec{p}_{2}-\vec{p}, \Omega_{2}\right) \bar{F}\left(\vec{p}_{2}, \Omega\right)+ \\
& \left.+W_{-}\left(\vec{p}-\vec{p}_{1}\right) \bar{F}\left(\vec{p}_{1}, \Omega\right) \bar{G}\left(\vec{p}-\vec{p}_{1}-\vec{p}_{2},-\Omega_{2}\right) \bar{F}\left(\vec{p}_{2}, \Omega_{2}\right)\right]+ \\
& +\frac{1}{V} \sum_{\vec{p}_{1}} W_{+}\left(\vec{p}-\vec{p}_{1}\right) \bar{G}\left(\vec{p}_{1}, \Omega\right), \\
& \Xi(\vec{p}, \Omega)=-\frac{1}{\beta V} \sum_{\vec{p}_{1}, \Omega_{1}} D\left(\vec{p}-\vec{p}_{1}, \Omega-\Omega_{1}\right) \bar{F}\left(\vec{p}_{1}, \Omega_{1}\right)+ \\
& +\frac{1}{(\beta V)^{2}} \sum_{\vec{p}_{1}, \Omega_{1}} \sum_{\vec{p}_{2}, \Omega_{2}} D\left(\vec{p}-\vec{p}_{1}, \Omega-\Omega_{1}\right) \times \\
& \times\left[D\left(\vec{p}-\vec{p}_{2}, \Omega-\Omega_{2}\right) \bar{G}\left(\vec{p}_{1}, \Omega_{1}\right) \bar{G}\left(\vec{p}_{1}+\vec{p}_{2}-\vec{p}, \Omega_{1}+\Omega_{2}-\Omega\right) \bar{F}\left(\vec{p}_{2}, \Omega_{2}\right)+\right. \\
& +D\left(\vec{p}_{1}-\vec{p}_{2}, \Omega_{1}-\Omega_{2}\right) \bar{G}\left(\vec{p}_{1}, \Omega_{1}\right) \bar{F}\left(\vec{p}_{2}, \Omega_{2}\right) \bar{G}\left(\vec{p}_{1}-\vec{p}-\vec{p}_{2}, \Omega_{1}-\Omega-\Omega_{2}\right)+ \\
& +D\left(\vec{p}-\vec{p}_{2}, \Omega-\Omega_{2}\right) \bar{F}\left(\vec{p}_{1}, \Omega_{1}\right) \bar{G}\left(\vec{p}-\vec{p}_{1}-\vec{p}_{2}, \Omega-\Omega_{1}-\Omega_{2}\right) \bar{G}\left(-\vec{p}_{2},-\Omega_{2}\right)- \\
& \left.-D\left(\vec{p}-\vec{p}_{2}, \Omega-\Omega_{2}\right) \bar{F}\left(\vec{p}_{1}, \Omega_{1}\right) \overline{\widetilde{F}}\left(\vec{p}_{2}+\vec{p}_{1}-\vec{p}, \Omega_{2}-\Omega_{1}-\Omega\right) \bar{F}\left(\vec{p}_{2}, \Omega_{2}\right)\right]+ \\
& +\frac{1}{\beta V^{2}} \sum_{\vec{p}_{1}, \Omega_{1}} \sum_{\vec{p}_{2}} D\left(\vec{p}-\vec{p}_{1}, \Omega-\Omega_{1}\right) \times \\
& \times\left[-W_{-}\left(\vec{p}_{1}-\vec{p}_{2}\right) \bar{G}\left(\vec{p}_{1}, \Omega_{1}\right) \bar{F}\left(\vec{p}_{2}, \Omega_{1}\right) \bar{G}\left(\vec{p}_{1}-\vec{p}_{2}-\vec{p},-\Omega\right)-\right. \\
& -W_{-}\left(\vec{p}-\vec{p}_{2}\right) \bar{G}\left(\vec{p}_{1}, \Omega_{1}\right) \bar{G}\left(\vec{p}_{1}+\vec{p}_{2}-\vec{p}, \Omega_{1}\right) \bar{F}\left(\vec{p}_{2}, \Omega\right)- \\
& -W_{+}\left(\vec{p}-\vec{p}_{2}\right) \bar{F}\left(\vec{p}_{1}, \Omega_{1}\right) \bar{G}\left(\vec{p}-\vec{p}_{1}-\vec{p}_{2},-\Omega_{1}\right) \bar{G}\left(-\vec{p}_{2},-\Omega\right)- \\
& \left.-W_{+}\left(\vec{p}-\vec{p}_{2}\right) \bar{F}\left(\vec{p}_{1}, \Omega_{1}\right) \overline{\widetilde{F}}\left(\vec{p}_{1}+\vec{p}_{2}-\vec{p}, \Omega_{1}\right) \bar{F}\left(\vec{p}_{2}, \Omega\right)\right]+ \\
& +\frac{1}{\beta V^{2}} \sum_{\vec{p}_{2}, \Omega_{2}} \sum_{\vec{p}_{1}}\left[-W_{+}\left(\vec{p}-\vec{p}_{1}\right) D\left(\vec{p}-\vec{p}_{2}, \Omega-\Omega_{2}\right) \times\right. \\
& \times \bar{G}\left(\vec{p}_{1}, \Omega\right) \bar{G}\left(\vec{p}_{1}+\vec{p}_{2}-\vec{p}, \Omega_{2}\right) \bar{F}\left(\vec{p}_{2}, \Omega_{2}\right)- \\
& -W_{-}\left(\vec{p}-\vec{p}_{1}\right) D\left(\vec{p}-\vec{p}_{2}, \Omega-\Omega_{2}\right) \bar{F}\left(\vec{p}_{1}, \Omega\right) \bar{G}\left(\vec{p}_{1}+\vec{p}_{2}-\vec{p}, \Omega_{2}\right) \bar{G}\left(\vec{p}_{2}, \Omega_{2}\right)- \\
& -W_{-}\left(\vec{p}-\vec{p}_{1}\right) D\left(\vec{p}_{1}-\vec{p}_{2}, \Omega_{1}-\Omega_{2}\right) \bar{G}\left(\vec{p}_{1}, \Omega\right) \bar{F}\left(\vec{p}_{2}, \Omega_{2}\right) \bar{G}\left(\vec{p}_{1}-\vec{p}_{2}-\vec{p},-\Omega_{2}\right)+ \\
& \left.+W_{+}\left(\vec{p}-\vec{p}_{1}\right) D\left(\vec{p}-\vec{p}_{2}, \Omega-\Omega_{2}\right) \bar{F}\left(\vec{p}_{1}, \Omega\right) \overline{\widetilde{F}}\left(\vec{p}_{1}+\vec{p}_{2}-\vec{p}, \Omega_{2}\right) \bar{F}\left(\vec{p}_{2}, \Omega_{2}\right)\right]+ \\
& +\frac{1}{V} \sum_{\vec{p}_{1}} W_{-}\left(\vec{p}-\vec{p}_{1}\right) \bar{F}\left(\vec{p}_{1}, \Omega\right),
\end{aligned}
$$


где

$$
W_{ \pm}\left(\vec{p}-\vec{p}_{1}\right)=\left|V_{1}\left(\vec{p}-\vec{p}_{1}\right)\right|^{2} \pm \frac{1}{4} S(S+1)\left|V_{2}\left(\vec{p}-\vec{p}_{1}\right)\right|^{2}
$$

\section{3. ОБЛАСТЬ ТЕМПЕРАТУР, БЛИЗКИХ К КРИТИЧЕСКОЙ}

При $T \sim T_{C}$ в выражениях (13) можно ограничиться линейным вкладом от операторов $\Xi(p)$ и представить функции Грина в виде

$$
\bar{G}(\vec{p}, \Omega)=\frac{1}{i \Omega-\varepsilon_{\vec{p}}-M_{N}(\vec{p}, \Omega)}, \quad F(\vec{p}, \Omega)=\bar{G}(-\vec{p},-\Omega) \Xi(\vec{p}, \Omega) \bar{G}(\vec{p}, \Omega),
$$

где

$$
\begin{aligned}
M_{N}(\vec{p}, \Omega)= & \frac{1}{\beta V} \sum_{\vec{p}_{1}, \Omega_{1}} \bar{V}_{N}\left(p, p_{1}\right) \bar{G}\left(\vec{p}_{1}, \Omega_{1}\right)+\frac{1}{V} \sum_{\vec{p}_{1}} \bar{W}_{+}\left(\vec{p}-\vec{p}_{1}\right) \bar{G}\left(\vec{p}_{1}, \Omega\right) \\
\Xi(\vec{p}, \Omega)= & \frac{1}{\beta V} \sum_{\vec{p}_{1}, \Omega_{1}} \bar{V}_{S}\left(p, p_{1}\right) \bar{F}\left(\vec{p}_{1}, \Omega_{1}\right)+\frac{1}{V} \sum_{\vec{p}_{1}} \bar{W}_{-}\left(\vec{p}-\vec{p}_{1}\right) \bar{F}\left(\vec{p}_{1}, \Omega\right) \\
\bar{V}_{N}\left(p, p_{1}\right)= & -D\left(\vec{p}-\vec{p}_{1}, \Omega-\Omega_{1}\right)+\frac{1}{\beta V} D\left(\vec{p}-\vec{p}_{1}, \Omega-\Omega_{1}\right) \sum_{\vec{p}_{2}, \Omega_{2}} D\left(\vec{p}-\vec{p}_{2}, \Omega-\Omega_{2}\right) \times \\
& \times \bar{G}\left(\vec{p}_{1}+\vec{p}_{2}-\vec{p}, \Omega_{1}+\Omega_{2}-\Omega\right) \bar{G}\left(\vec{p}_{2}, \Omega_{2}\right), \\
\bar{V}_{S}\left(p, p_{1}\right)= & -D\left(\vec{p}-\vec{p}_{1}, \Omega-\Omega_{1}\right)+ \\
& +\frac{1}{\beta V} \sum_{\vec{p}_{2}, \Omega_{2}}\left\{D\left(\vec{p}-\vec{p}_{1}, \Omega-\Omega_{1}\right) D\left(\vec{p}-\vec{p}_{2}, \Omega-\Omega_{2}\right) \times\right. \\
& \times \bar{G}\left(\vec{p}_{2}, \Omega_{2}\right) \bar{G}\left(\vec{p}_{1}+\vec{p}_{2}-\vec{p}, \Omega_{1}+\Omega_{2}-\Omega\right)+ \\
& +D\left(\vec{p}_{2}-\vec{p}_{1}, \Omega_{2}-\Omega_{1}\right) D\left(\vec{p}-\vec{p}_{2}, \Omega-\Omega_{2}\right) \times \\
& \times \bar{G}\left(\vec{p}_{2}, \Omega_{2}\right) \bar{G}\left(\vec{p}_{2}-\vec{p}-\vec{p}_{1}, \Omega_{2}-\Omega-\Omega_{1}\right)+ \\
& +D\left(\vec{p}-\vec{p}_{1}, \Omega-\Omega_{1}\right) D\left(\vec{p}-\vec{p}_{2}, \Omega-\Omega_{2}\right) \times \\
& \left.\times \bar{G}\left(\vec{p}-\vec{p}_{1}-\vec{p}_{2}, \Omega-\Omega_{1}-\Omega_{2}\right) \bar{G}\left(-\vec{p}_{2},-\Omega_{2}\right)\right\}- \\
& -\frac{1}{V} \sum_{\vec{p}_{2}}\left\{W_{-}\left(\vec{p}_{2}-\vec{p}_{1}\right) D\left(\vec{p}-\vec{p}_{2}, \Omega-\Omega_{1}\right) \times\right. \\
& \times \bar{G}\left(\vec{p}_{2}, \Omega_{1}\right) \bar{G}\left(\vec{p}_{2}-\vec{p}_{1}-\vec{p},-\Omega\right)+ \\
& +W+\left(\vec{p}-\vec{p}_{2}\right) D\left(\vec{p}-\vec{p}_{1}, \Omega-\Omega_{1}\right) \bar{G}\left(\vec{p}-\vec{p}_{1}-\vec{p}_{2},-\Omega_{1}\right) \bar{G}\left(-\vec{p}_{2},-\Omega\right)+ \\
& +W_{+}\left(\vec{p}-\vec{p}_{2}\right) D\left(\vec{p}-\vec{p}_{1}, \Omega-\Omega_{1}\right) \bar{G}\left(\vec{p}_{2}, \Omega\right) \bar{G}\left(\vec{p}_{1}+\vec{p}_{2}-\vec{p}, \Omega_{1}\right)+ \\
& \left.+W-\left(\vec{p}-\vec{p}_{2}\right) D\left(\vec{p}_{2}-\vec{p}_{1}, \Omega-\Omega_{1}\right) \bar{G}\left(\vec{p}_{2}, \Omega\right) \bar{G}\left(\vec{p}_{2}-\vec{p}_{1}-\vec{p},-\Omega_{1}\right)\right\} \\
\bar{W}_{ \pm}\left(\vec{p}-\vec{p}_{1}\right)= & W_{ \pm}\left(\vec{p}-\vec{p}_{1}\right) \times \\
\times\{ & \left.1-\frac{2}{\beta V} \sum_{\vec{p}_{2}, \Omega_{2}} D\left(\vec{p}-\vec{p}_{2}, \Omega-\Omega_{2}\right) \bar{G}\left(\vec{p}_{2}, \Omega_{2}\right) \bar{G}\left(\vec{p}_{1}+\vec{p}_{2}-\vec{p}, \Omega_{2}\right)\right\}
\end{aligned}
$$


Выполнив в уравнениях (19) и (20) усреднение по поверхности Ферми, запишем их в виде

$$
\begin{aligned}
M_{N}(\Omega)= & \frac{1}{\beta V} \sum_{\vec{p}_{1}, \Omega_{1}} \bar{V}_{N}\left(\Omega \Omega_{1}\right) \bar{G}\left(\vec{p}_{1}, \Omega_{1}\right)+\frac{1}{V} \sum_{\vec{p}_{1}} \bar{W}_{+} \bar{G}\left(\vec{p}_{1}, \Omega\right), \\
\Xi(\Omega)= & \frac{1}{\beta V} \sum_{\vec{p}_{1}, \Omega_{1}} \bar{V}_{S}\left(\Omega \Omega_{1}\right) \bar{G}\left(\vec{p}_{1}, \Omega_{1}\right) \Xi\left(\Omega_{1}\right) \bar{G}\left(-\vec{p}_{1},-\Omega_{1}\right)+ \\
& +\frac{1}{V} \sum_{\vec{p}_{1}} \bar{W}_{-} \bar{G}\left(\vec{p}_{1}, \Omega\right) \Xi(\Omega) \bar{G}\left(-\vec{p}_{1},-\Omega\right),
\end{aligned}
$$

где $\bar{V}_{N}\left(\Omega \Omega_{1}\right), \bar{V}_{S}\left(\Omega \Omega_{1}\right)$ и $\bar{W}_{ \pm}$определяются выражениями $(21)-(23)$, также усредненными по поверхности Ферми. Выполним далее в $(24),(25)$ интегрирование по энергии в пределах $-E / 2<\varepsilon_{\vec{p}_{1}}<E / 2$ обычным способом и введем определения

$$
\begin{aligned}
& \widetilde{\Omega}=\widetilde{\Omega}(\Omega)=\Omega-\operatorname{Im} M_{N}(\Omega)= \\
& =\Omega+\frac{2 \pi N_{0}}{\beta} \sum_{\Omega_{1}} \bar{V}_{N}\left(\Omega \Omega_{1}\right) \frac{\widetilde{\Omega}_{1}}{\pi\left|\widetilde{\Omega}_{1}\right|} \operatorname{arctg} \frac{E}{2\left|\widetilde{\Omega}_{1}\right|}+ \\
& +2 \pi N_{0} \bar{W}_{+} \frac{\widetilde{\Omega}}{\pi|\widetilde{\Omega}|} \operatorname{arctg} \frac{E}{2|\widetilde{\Omega}|} \\
& \Xi(\Omega)=\tilde{\Delta}(\Omega)=\bar{\Delta}+2 \pi N_{0} \bar{W}_{-} \frac{\tilde{\Delta}(\Omega)}{\pi|\widetilde{\Omega}|} \operatorname{arctg} \frac{E}{2|\widetilde{\Omega}|}, \\
& \bar{\Delta}=\frac{2 \pi N_{0}}{\beta} \sum_{\Omega_{1}} \bar{V}_{S}\left(\Omega \Omega_{1}\right) \frac{\tilde{\Delta}\left(\Omega_{1}\right)}{\pi\left|\widetilde{\Omega}_{1}\right|} \operatorname{arctg} \frac{E}{2\left|\widetilde{\Omega}_{1}\right|} .
\end{aligned}
$$

Самосогласованная система уравнений (26)-(28) определяет температуру сверхпроводящего перехода в рассматриваемой примесной системе. Необходимо рассмотреть выражения $\bar{V}_{N}, \bar{V}_{S}$, а также $\bar{W}_{ \pm}$. Следуя [5], воспользуемся представлением

$$
D\left(\vec{p}-\vec{p}_{1}, \Omega-\Omega_{1}\right)=\left|g_{\vec{p} \vec{p}_{1}}\right|^{2} D\left(\Omega, \Omega_{1}\right)=g^{2}\left(\frac{2 k_{\mathrm{F}}}{q_{c}}\right)^{2} \theta\left(q_{c}-\left|\vec{p}-\vec{p}_{1}\right|\right) D\left(\Omega, \Omega_{1}\right)
$$

и введем модель для примесного взаимодействия

$$
\begin{aligned}
W_{ \pm}\left(\vec{p}-\vec{p}_{1}\right) & =W_{ \pm}\left(\frac{2 k_{\mathrm{F}}}{q_{c 1}}\right)^{2} \theta\left(q_{c 1}-\left|\vec{p}-\vec{p}_{1}\right|\right) \\
W_{ \pm} & =V_{1}^{2}+\frac{1}{4} S(S+1) V_{2}^{2}
\end{aligned}
$$

где $q_{c}$ и $q_{c 1}$ - максимальные значения передаваемого импульса при взаимодействии электронов с фононами и с примесью, соответственно. Для фононного пропагатора предполагаем простой эйнштейновский спектр с частотой $\omega_{0}$ :

$$
D\left(\Omega, \Omega_{1}\right)=-\frac{\omega_{0}^{2}}{\Omega^{2}+\omega_{0}^{2}}
$$


Введем определения

$$
\begin{aligned}
\bar{P}_{V}\left(\vec{p} \vec{p}_{1}, \Omega \Omega_{1}\right)= & -\frac{1}{\beta V N_{0}}\left(\frac{2 k_{\mathrm{F}}}{q_{c}}\right)^{2} \sum_{\vec{p}_{2}, \Omega_{2}} \theta\left(q_{c}-\left|\vec{p}-\vec{p}_{2}\right|\right) \times \\
& \times \bar{G}\left(\vec{p}_{2}, \Omega_{2}\right) \bar{G}\left(\vec{p}_{1}+\vec{p}_{2}-\vec{p}, \Omega_{1}+\Omega_{2}-\Omega\right) D\left(\Omega, \Omega_{2}\right), \\
\bar{P}_{c}\left(\vec{p} \vec{p}_{1}, \Omega \Omega_{1}\right)= & -\frac{1}{\beta V N_{0}}\left(\frac{2 k_{\mathrm{F}}}{q_{c}}\right)^{2} \sum_{\vec{p}_{2}, \Omega_{2}} \theta\left(q_{c}-\left|\vec{p}-\vec{p}_{2}\right|\right) \times \\
& \times \bar{G}\left(\vec{p}_{2}, \Omega_{2}\right) \bar{G}\left(\vec{p}_{2}-\vec{p}-\vec{p}_{1}, \Omega_{2}-\Omega-\Omega_{1}\right) D\left(\Omega, \Omega_{2}\right)
\end{aligned}
$$

и

$$
\begin{aligned}
\Gamma_{V}\left(\vec{p} \vec{p}_{1}, \Omega \Omega_{1}\right)= & W_{+}\left(\frac{2 k_{\mathrm{F}}}{q_{c 1}}\right)^{2} \frac{1}{N_{0} V} \sum_{\vec{p}_{2}} \theta\left(q_{c 1}-\left|\vec{p}-\vec{p}_{2}\right|\right) \bar{G}\left(\vec{p}_{2}, \Omega\right) \bar{G}\left(\vec{p}_{1}+\vec{p}_{2}-\vec{p}, \Omega_{1}\right) \\
\Gamma_{c}^{\mathrm{I}}\left(\vec{p} \vec{p}_{1}, \Omega \Omega_{1}\right)= & W_{-}\left(\frac{2 k_{\mathrm{F}}}{q_{c}}\right)^{2} \frac{1}{N_{0} V} \sum_{\vec{p}_{2}} \theta\left(q_{c}-\left|\vec{p}-\vec{p}_{2}\right|\right) \bar{G}\left(\vec{p}_{2}, \Omega_{1}\right) \bar{G}\left(\vec{p}_{2}-\vec{p}_{1}-\vec{p},-\Omega\right) \\
\Gamma_{c}^{\mathrm{II}}\left(\vec{p} \vec{p}_{1}, \Omega \Omega_{1}\right)= & W_{+}\left(\frac{2 k_{\mathrm{F}}}{q_{c 1}}\right)^{2} \frac{1}{N_{0} V} \sum_{\vec{p}_{2}} \theta\left(q_{c 1}-\left|\vec{p}-\vec{p}_{2}\right|\right) \times \\
& \times \bar{G}\left(\vec{p}_{2}, \Omega\right) \bar{G}\left(\vec{p}_{2}-\vec{p}_{1}-\vec{p},-\Omega_{1}\right) .
\end{aligned}
$$

Величины $P_{V}$ и $P_{c}$ появляются вследствие учета электрон-фононного взаимодействия, а $\Gamma_{V}$ и $\Gamma_{c}-$ примесного. На основании определений (21)-(23), а также определений (33), (34) имеем

$$
\begin{aligned}
\bar{V}_{N}\left(p p_{1}\right)= & -g^{2} D\left(\Omega \Omega_{1}\right)\left(\frac{2 k_{\mathrm{F}}}{q_{c}}\right)^{2} \theta\left(q_{c}-\left|\vec{p}-\vec{p}_{1}\right|\right)\left[1+\lambda \bar{P}_{V}\left(\vec{p} \vec{p}_{1}, \Omega \Omega_{1}\right)\right] \\
\bar{V}_{S}\left(p p_{1}\right)= & -g^{2} D\left(\Omega \Omega_{1}\right)\left(\frac{2 k_{\mathrm{F}}}{q_{c}}\right)^{2} \theta\left(q_{c}-\left|\vec{p}-\vec{p}_{1}\right|\right) \times \\
& \times\left[1+2 \lambda \bar{P}_{V}\left(\vec{p}_{1}, \Omega \Omega_{1}\right)+\lambda \bar{P}_{c}\left(\vec{p} \vec{p}_{1}, \Omega \Omega_{1}\right)\right]- \\
& -\lambda D\left(\Omega \Omega_{1}\right)\left(\frac{2 k_{\mathrm{F}}}{q_{c 1}}\right)^{2} \theta\left(q_{c 1}-\left|\vec{p}-\vec{p}_{1}\right|\right) \Gamma_{c}^{\mathrm{I}}\left(\vec{p} \vec{p}_{1}, \Omega \Omega_{1}\right)- \\
& -\lambda D\left(\Omega \Omega_{1}\right)\left(\frac{2 k_{\mathrm{F}}}{q_{c}}\right)^{2} \theta\left(q_{c}-\left|\vec{p}-\vec{p}_{1}\right|\right) \Gamma_{c}^{\mathrm{II}}\left(\vec{p} \vec{p}_{1}, \Omega \Omega_{1}\right)- \\
& -2 \lambda D\left(\Omega \Omega_{1}\right)\left(\frac{2 k_{\mathrm{F}}}{q_{c}}\right)^{2} \theta\left(q_{c}-\left|\vec{p}-\vec{p}_{1}\right|\right) \Gamma_{V}\left(\vec{p} \vec{p}_{1}, \Omega \Omega_{1}\right) \\
\bar{W}_{ \pm}\left(\vec{p}-\vec{p}_{1}\right)= & W_{ \pm}\left(\frac{2 k_{\mathrm{F}}}{q_{c}}\right)^{2} \theta\left(q_{c 1}-\left|\vec{p}-\vec{p}_{1}\right|\right)\left[1+2 \lambda \bar{P}_{V}\left(\vec{p} \vec{p}_{1}, \Omega \Omega_{1}\right)\right]
\end{aligned}
$$


Величины $\bar{P}_{V}$ и $\bar{P}_{c}$ слабо отличаются от своих значений $P_{V}$ и $P_{c}$ для случая чистого сверхпроводника, детально рассмотренных в [5]. Вычисление же величин $\Gamma_{V}$ и $\Gamma_{c}$ приведено в приложении. Имеем

$$
\begin{aligned}
\Gamma_{V}\left(\vec{p} \vec{p}_{1}, \Omega \Omega_{1}\right)= & W_{+}\left\{L\left(\Omega \Omega_{1}\right)+\frac{1}{2 E^{2} Q^{2} Q_{c 1}^{2}}\left[K\left(\Omega \Omega_{1}\right)-\left(\Omega-\Omega_{1}\right)^{2} L\left(\Omega \Omega_{1}\right)\right] \times\right. \\
& \left.\times\left[\sqrt{1+\left(\frac{2 E Q Q_{c 1}}{\Omega-\Omega_{1}}\right)^{2}}-1\right]\right\}, \\
\Gamma_{c}^{\mathrm{I}}\left(\vec{p} \vec{p}_{1}, \Omega \Omega_{1}\right)= & W_{-}\left\{L\left(-\Omega_{1} \Omega\right)+\frac{1}{2 E^{2} Q_{c}^{2}\left(1-Q^{2}\right)} \times\right. \\
& \times\left[\frac{K\left(-\Omega_{1} \Omega\right)}{\Omega+\Omega_{1}}-\left(\Omega+\Omega_{1}\right) L\left(-\Omega_{1} \Omega\right)\right] \times \\
& \left.\times \operatorname{arctg} \frac{2 Q_{c}^{2} E\left(1-Q^{2}\right)}{\Omega+\Omega_{1}}\right\}, \\
\Gamma_{c}^{\mathrm{II}}\left(\vec{p} \vec{p}_{1}, \Omega \Omega_{1}\right)= & W_{-}\left\{L\left(-\Omega_{1}\right)+\frac{1}{2 E^{2} Q_{c 1}^{2}\left(1-Q^{2}\right)} \times\right. \\
& \times\left[\frac{K\left(-\Omega_{1}\right)}{\Omega+\Omega_{1}}-\left(\Omega+\Omega_{1}\right) L\left(-\Omega_{1}\right)\right] \times \\
& \left.\times \operatorname{arctg} \frac{2 Q_{c 1}^{2} E\left(1-Q^{2}\right)}{\Omega+\Omega_{1}}\right\},
\end{aligned}
$$

где

$$
\begin{aligned}
K\left(\Omega \Omega_{1}\right) & =2\left(\Omega-\Omega_{1}\right)\left[\operatorname{arctg} \frac{E}{2 \Omega}-\operatorname{arctg} \frac{E}{2 \Omega_{1}}\right] \\
L\left(\Omega \Omega_{1}\right) & =-\frac{E}{\frac{E^{2}}{4}+\Omega_{1}^{2}}+\frac{\Omega-\Omega_{1}}{\Omega_{1}^{3}} \frac{E}{\left[1+\left(\frac{E}{2 \Omega_{1}}\right)^{2}\right]^{2}}, \\
Q_{c} & =\frac{q_{c}}{2 k_{\mathrm{F}}}, \quad Q=\frac{q}{2 k_{\mathrm{F}}} .
\end{aligned}
$$

Полагая поверхность Ферми сферической, выполним в (35)-(37) угловое усреднение согласно формуле [5]

$$
\begin{gathered}
\left(\frac{2 k_{\mathrm{F}}}{q_{c}}\right)^{2} \int \frac{d \Omega_{p}}{4 \pi} \int \frac{d \Omega_{p 1}}{4 \pi} \theta\left(q_{c}-\left|\vec{p}-\vec{p}_{1}\right|\right) \bar{P}_{V}\left(\vec{p} \vec{p}_{1}, \Omega \Omega_{1}\right)= \\
=\frac{2}{Q_{c}^{2}} \int_{0}^{Q_{c}} d Q Q \bar{P}_{V}\left(Q Q_{c}, \Omega \Omega_{1}\right)=P_{V}\left(Q_{c}, \Omega \Omega_{1}\right) .
\end{gathered}
$$

Получаем

$$
\begin{aligned}
& \bar{V}_{N}\left(\Omega \Omega_{1}\right)=-g^{2} D\left(\Omega \Omega_{1}\right)\left[1+\lambda P_{V}\left(Q_{c}, \Omega \Omega_{1}\right)\right] \\
& \bar{V}_{S}\left(\Omega \Omega_{1}\right)=-g^{2} D\left(\Omega \Omega_{1}\right)\left[1+2 \lambda P_{V}\left(Q_{c}, \Omega \Omega_{1}\right)+\lambda P_{c}\left(Q_{c}, \Omega \Omega_{1}\right)-\lambda D\left(\Omega \Omega_{1}\right)\right] \times
\end{aligned}
$$




$$
\begin{aligned}
& \times\left[\Gamma_{c}^{\mathrm{I}}\left(Q_{c} Q_{c 1}, \Omega \Omega_{1}\right)+\Gamma_{c}^{\mathrm{II}}\left(Q_{c} Q_{c 1}, \Omega \Omega_{1}\right)+2 \Gamma_{V}\left(Q_{c} Q_{c 1}, \Omega \Omega_{1}\right)\right] \\
\bar{W}_{ \pm}= & W_{ \pm}\left[1+2 \lambda P_{V}(\Omega \Omega)\right]
\end{aligned}
$$

где

$$
P_{V}(\Omega \Omega)=\left.P_{V}\left(Q_{c}, \Omega \Omega_{1}\right)\right|_{\Omega_{1}=\Omega} .
$$

Выражения для $P_{V}$ и $P_{c}$ даны в [5], здесь мы приведем их значения при $\Omega=0$ и $\Omega_{1}=\omega_{0}$. Имеем

$$
\begin{aligned}
P_{V}\left(m, Q_{c}\right)= & -\varphi(m)+\left[\frac{\pi}{4}-\operatorname{arctg} \frac{m}{1+m}+\varphi(m)\right] \times \\
& \times \frac{m^{2}}{4 Q_{c}^{4}}\left\{\sqrt{\eta_{c}(m)}-1-\ln \frac{1+\sqrt{\eta_{c}(m)}}{2}\right\}, \\
P_{c}\left(m, Q_{c}\right)= & -\varphi(m)+\left[\frac{\pi}{4}-\operatorname{arctg} \frac{m}{1+m}+\varphi(m)\right] \times \\
& \times \frac{m}{4 Q_{c}^{2}\left(1-\frac{Q_{c}^{2}}{2}\right)} \operatorname{arctg} \frac{4 Q_{c}^{2}\left(1-\frac{Q_{c}^{2}}{2}\right)}{m},
\end{aligned}
$$

где

$$
\begin{gathered}
\varphi(m)=m(1+m) \frac{(1+m)^{2}+2 m^{2}}{\left[(1+m)^{2}+m^{2}\right]^{2}}, \quad \eta_{c}(m)=1+\left(\frac{4 Q_{c}^{2}}{m}\right)^{2} \\
\left.P_{V}(\Omega \Omega)\right|_{\Omega=0}=-\frac{m}{1+m} .
\end{gathered}
$$

Здесь $m=2 \omega_{0} / E$ - параметр Мигдала.

Выражения же для вершинных функций смешанного типа после усреднения по поверхности Ферми запишутся в виде

$$
\begin{aligned}
\Gamma_{V}\left(Q_{c} Q_{c 1}, \Omega \Omega_{1}\right)= & W_{+}\left\{L\left(\Omega \Omega_{1}\right)+\left[K\left(\Omega \Omega_{1}\right)-\left(\Omega-\Omega_{1}\right)^{2} L\left(\Omega \Omega_{1}\right)\right] \times\right. \\
& \times \frac{1}{E^{2} Q_{c}^{2} Q_{c 1}^{2}}\left[\sqrt{1+\left(\frac{2 E Q_{c} Q_{c 1}}{\Omega-\Omega_{1}}\right)^{2}}-\right. \\
& \left.\left.-1-\ln \frac{1}{2}\left(1+\sqrt{1+\left(\frac{2 E Q_{c} Q_{c 1}}{\Omega-\Omega_{1}}\right)^{2}}\right)\right]\right\}, \\
\Gamma_{c}^{\mathrm{I}}\left(Q_{c} Q_{c 1}, \Omega \Omega_{1}\right)= & W_{-}\left\{L\left(-\Omega_{1} \Omega\right)+\left[\frac{K\left(-\Omega_{1} \Omega\right)}{\Omega+\Omega_{1}}-\left(\Omega+\Omega_{1}\right) L\left(-\Omega_{1} \Omega\right)\right] \times\right. \\
& \left.\times \frac{1}{2 E Q_{c}^{2} Q_{c 1}^{2}} \int_{0}^{Q_{c 1}} \frac{Q d Q}{1-Q^{2}} \operatorname{arctg} \frac{2 Q_{c}^{2} E\left(1-Q^{2}\right)}{\Omega+\Omega_{1}}\right\},
\end{aligned}
$$

а выражение для $\Gamma_{c}^{\mathrm{II}}$ получим из (51), заменив $\Omega \leftrightarrow \Omega_{1}, Q_{c} \leftrightarrow Q_{c 1}$. 
Самосогласованная система уравнений (26)-(28) с учетом (43)-(45) приобретает вид

$$
\begin{aligned}
\bar{\Delta}= & \frac{2 \pi}{\beta} \lambda \sum_{\Omega_{1}} \frac{\omega_{0}^{2}}{\left(\Omega-\Omega_{1}\right)^{2}+\omega_{0}^{2}}\left[1+2 \lambda P_{V}\left(Q_{c}, \Omega \Omega_{1}\right)+\lambda P_{c}\left(Q_{c}, \Omega \Omega_{1}\right)+\right. \\
& +\Gamma_{c}^{\mathrm{I}}\left(Q_{c} Q_{c 1}, \Omega \Omega_{1}\right)+\Gamma_{c}^{\mathrm{II}}\left(Q_{c} Q_{c 1}, \Omega \Omega_{1}\right)+ \\
& \left.+2 \Gamma_{V}\left(Q_{c} Q_{c 1}, \Omega \Omega_{1}\right)\right] \frac{\tilde{\Delta}\left(\Omega_{1}\right)}{\pi\left|\widetilde{\Omega}_{1}\right|} \operatorname{arctg} \frac{E}{2\left|\widetilde{\Omega}_{1}\right|}, \\
\tilde{\Delta}(\Omega)= & \bar{\Delta}(\Omega)+2 \pi N_{0} W_{-}\left[1+2 \lambda P_{V}(\Omega \Omega)\right] \frac{\tilde{\Delta}(\Omega)}{\pi|\widetilde{\Omega}|} \operatorname{arctg} \frac{E}{2|\widetilde{\Omega}|}, \\
\widetilde{\Omega}= & +\frac{2 \pi}{\beta} \lambda \sum_{\Omega_{1}} \frac{\omega_{0}^{2}}{\left(\Omega-\Omega_{1}\right)^{2}+\omega_{0}^{2}}\left[1+\lambda P_{V}\left(Q_{c}, \Omega \Omega_{1}\right)\right] \operatorname{arctg} \frac{E}{2 \widetilde{\Omega}_{1}}+ \\
& +2 \pi N_{0} W_{+}\left[1+2 \lambda P_{V}(\Omega \Omega)\right] \frac{1}{\pi} \operatorname{arctg} \frac{E}{2 \widetilde{\Omega}} .
\end{aligned}
$$

\section{4. ТЕМПЕРАТУРА СВЕРХПРОВОДЯЩЕГО ПЕРЕХОДА И ИЗОТОПИЧЕСКИЙ ЭФФЕКТ}

Проанализируем полученные в предыдущем разделе уравнения (52)-(54) для того, чтобы выяснить совместное влияние эффекта неадиабатичности и парамагнитной примеси на температуру сверхпроводящего перехода $T_{C}$ и изотопический коэффициент $\alpha . \mathrm{C}$ этой целью выполним ряд упрошений. Положим в квадратных скобках в этих уравнениях $\Omega=0, \Omega_{1}=\omega_{0}$ и рассмотрим приближение слабой связи $T_{C} / \omega_{0} \ll 1$. В соответствии с этим введем обозначения

$$
\begin{aligned}
\lambda_{\Delta}= & \lambda\left[1+2 \lambda P_{V}\left(m, Q_{c}\right)+\lambda P_{c}\left(m, Q_{c}\right)+\right. \\
& \left.+\Gamma_{c}^{\mathrm{I}}\left(Q_{c} Q_{c 1}, \omega_{0}\right)+\Gamma_{c}^{\mathrm{II}}\left(Q_{c} Q_{c 1}, \omega_{0}\right)+\Gamma_{V}\left(Q_{c} Q_{c 1}, \omega_{0}\right)\right], \\
\lambda_{z}= & \lambda\left[1+2 \lambda P_{V}\left(m, Q_{c}\right)\right], \quad \bar{W}_{ \pm}=W_{ \pm}\left[1+2 \lambda P_{V}(\Omega, \Omega)_{\Omega=0}\right] .
\end{aligned}
$$

На основании формул $(50),(51)$ и (41) нетрудно видеть, что $\Gamma_{V, c}$ в этом приближении оказываются порядка $W_{ \pm} / E \ll 1$, что позволяет в дальнейшем пренебречь этими членами в (55) и рассматривать величину $\lambda_{\Delta}$ как не зависящую от концентрации примеси. Учитывая этот факт, используем определения (55) и приведем уравнения (52)-(54) к виду

$$
\begin{aligned}
\bar{\Delta}(\Omega) & =\frac{2 \pi}{\beta} \lambda_{\Delta}^{0} \sum_{\Omega_{1}} \frac{\omega_{0}^{2}}{\left(\Omega-\Omega_{1}\right)^{2}+\omega_{0}^{2}} \frac{\tilde{\Delta}\left(\Omega_{1}\right)}{\pi\left|\widetilde{\Omega}_{1}\right|} \operatorname{arctg} \frac{E}{2\left|\widetilde{\Omega}_{1}\right|}, \\
\tilde{\Delta}(\Omega) & =\bar{\Delta}(\Omega)+2 \pi N_{0} \bar{W}_{-} \frac{\tilde{\Delta}(\Omega)}{\pi|\widetilde{\Omega}|} \operatorname{arctg} \frac{E}{2|\widetilde{\Omega}|}, \\
\widetilde{\Omega} & =\Omega Z_{0}+2 \pi N_{0} \bar{W}_{+} \frac{1}{\pi} \operatorname{arctg} \frac{E}{2 \widetilde{\Omega}},
\end{aligned}
$$


где

$$
Z_{0}=1+\frac{2 \pi}{\beta \Omega} \lambda_{z} \sum_{\Omega_{1}} \frac{\omega_{0}^{2}}{\left(\Omega-\Omega_{1}\right)^{2}+\omega_{0}^{2}} \operatorname{arctg} \frac{E}{2 \widetilde{\Omega}_{1}}=1+\frac{\lambda_{z}}{1+m}, \quad \lambda_{\Delta}^{0}=\left.\lambda_{\Delta}\right|_{c=0} .
$$

Введем величину $u=\widetilde{\Omega} / \tilde{\Delta}$ и приведем последние два из уравнений $(56)$ к виду

$$
u(\Omega)=\frac{\Omega Z_{0}}{\bar{\Delta}}+\frac{2 \pi N_{0}}{\bar{\Delta}}\left[\bar{W}_{+}-\bar{W}_{-}\right] \frac{1}{\pi} \operatorname{arctg} \frac{E}{2 \widetilde{\Omega}} .
$$

Имеем также

$$
\bar{\Delta}(\Omega)=\frac{2 \pi}{\beta} \lambda_{\Delta}^{0} \sum_{\Omega_{1}} \frac{\omega_{0}^{2}}{\left(\Omega-\Omega_{1}\right)^{2}+\omega_{0}^{2}} \frac{1}{\pi\left|u\left(\Omega_{1}\right)\right|} \operatorname{arctg} \frac{E}{2\left|\widetilde{\Omega}_{1}\right|} .
$$

Поскольку в (57) функция под знаком суммы быстро сходится, можно заменить $u\left(\Omega_{1}\right)$ его значением в области малых $\Omega_{1}$, т.е. положить

$$
u\left(\Omega_{1}\right)=\frac{\Omega_{1} Z_{0}}{\bar{\Delta}}+\frac{\pi N_{0}}{\bar{\Delta}}\left[\bar{W}_{+}-\bar{W}_{-}\right] \operatorname{sign} \Omega_{1}
$$

С учетом (59) уравнение для параметра порядка $\bar{\Delta}$ приведем к виду

$$
\bar{\Delta}(\Omega)=\frac{2 \pi}{\beta} \lambda_{\Delta}^{0} \sum_{\Omega_{1}} \frac{\omega_{0}^{2}}{\left(\Omega-\Omega_{1}\right)^{2}+\omega_{0}^{2}} \frac{\bar{\Delta}\left(\Omega_{1}\right)}{Z_{0} \Omega_{1}+\pi N_{0}\left[\bar{W}_{+}-\bar{W}_{-}\right] \operatorname{sign} \Omega_{1}} \frac{1}{\pi} \operatorname{arctg} \frac{E}{2 \widetilde{\Omega}_{1}} .
$$

При получении этого уравнения мы пренебрегаем вкладами порядка $W_{ \pm} / E \ll 1$. Далее выполним преобразования, аналогичные случаю адиабатической теории [10] (см. также [5]). Ядро уравнения (60) представим в виде

$$
\frac{\omega_{0}^{2}}{\left(\Omega-\Omega_{1}\right)^{2}+\omega_{0}^{2}} \rightarrow \frac{\omega_{0}^{2}}{\Omega^{2}+\omega_{0}^{2}} \frac{\omega_{0}^{2}}{\Omega_{1}^{2}+\omega_{0}^{2}}
$$

Подставим это выражение в (60) и введем определение

$$
A=\frac{2 \pi}{\beta} \lambda_{\Delta}^{0} \sum_{\Omega_{1}} \frac{\omega_{0}^{2}}{\Omega_{1}^{2}+\omega_{0}^{2}} \frac{\bar{\Delta}\left(\Omega_{1}\right)}{Z_{0} \Omega_{1}+\pi N_{0}\left[\bar{W}_{+}-\bar{W}_{-}\right] \operatorname{sign} \Omega_{1}} \frac{1}{\pi} \operatorname{arctg} \frac{E}{2 \widetilde{\Omega}_{1}}
$$

rorra

$$
\bar{\Delta}(\Omega)=\frac{\omega_{0}^{2}}{\Omega^{2}+\omega_{0}^{2}} A .
$$

Подставляя в правую часть (62) выражение (63), получаем уравнение для определения температуры сверхпроводяшего перехода

$$
\frac{Z_{0}}{\lambda_{\Delta}^{0}}=\pi T_{C} \sum_{\Omega_{1}} \frac{\omega_{0}^{4}}{\left(\Omega_{1}^{2}+\omega_{0}^{2}\right)^{2}} \frac{1}{\Omega_{1}+\bar{\gamma} \operatorname{sign} \omega_{1}} \frac{2}{\pi} \operatorname{arctg} \frac{E}{2 \Omega_{1}}=
$$




$$
\begin{aligned}
= & 2 \pi T_{C}\left\{\sum_{\Omega_{1}>0} \frac{\omega_{0}^{2}}{\Omega_{1}^{2}+\omega_{0}^{2}} \frac{1}{\Omega_{1}+\bar{\gamma}}-\sum_{\Omega_{1}>0} \frac{\omega_{0}^{2}}{\Omega_{1}^{2}+\omega_{0}^{2}} \frac{1}{\Omega_{1}+\bar{\gamma}} \frac{2}{\pi} \operatorname{arctg} \frac{2 \Omega_{1}}{E}-\right. \\
& \left.-\sum_{\Omega_{1}>0} \frac{\omega_{0}^{2} \Omega_{1}^{2}}{\left(\Omega_{1}^{2}+\omega_{0}^{2}\right)^{2}} \frac{1}{\Omega_{1}+\bar{\gamma}}+\sum_{\Omega_{1}>0} \frac{\omega_{0}^{2} \Omega_{1}^{2}}{\left(\Omega_{1}^{2}+\omega_{0}^{2}\right)^{2}} \frac{1}{\Omega_{1}+\bar{\gamma}} \frac{2}{\pi} \operatorname{arctg} \frac{2 \Omega_{1}}{E}\right\},
\end{aligned}
$$

где

$$
\bar{\gamma}=\frac{\pi N_{0}}{Z_{0}}\left[\bar{W}_{+}-\bar{W}_{-}\right]
$$

Вклад первой из сумм в (64) может быть приведен к виду

$$
2 \pi T_{C} \sum_{\Omega_{1}>0} \frac{\omega_{0}^{2}}{\Omega_{1}^{2}+\omega_{0}^{2}} \frac{1}{\Omega_{1}+\bar{\gamma}}=\operatorname{Re} \psi\left(\frac{1}{2}+i \frac{\omega_{0}}{2 \pi T_{C}}\right)-\psi\left(\frac{1}{2}+\rho\right)
$$

где

$$
\begin{gathered}
\rho=\frac{\Gamma}{2 \pi T_{C}} f_{c}(m), \\
f_{c}(m)=\frac{1}{Z_{0}}\left(1-2 \lambda \frac{m}{1+m}\right), \quad \Gamma=\frac{1}{2 \tau_{s}}=\frac{1}{2} c N_{0} \pi S(1+S),
\end{gathered}
$$

$\psi$ - диаграмма-функция, $\tau_{s}$ - время релаксации рассеяния на магнитной примеси. Другие суммы в (64) не содержат логарифмической особенности по температуре, и в приближении слабой связи $\left(T_{C} / \omega_{0} \ll 1\right)$ в них можно перейти к пределу $T_{C} \rightarrow 0$, выполнив интегрирование по $\Omega_{1}$ обычным способом.

В результате этих вычислений, отбрасывая члены порядка $\Gamma / E \ll 1$, для температуры сверхпроводяшего перехода $T_{C}$ получаем

$$
\ln \frac{T_{C}}{T_{C 0}}=\psi\left(\frac{1}{2}\right)-\psi\left(\frac{1}{2}+\rho\right)
$$

Здесь

$$
T_{C 0}=\frac{2 \omega_{0} \gamma_{\ni}}{\sqrt{e}(1+m) \pi} \exp \left[\frac{1}{2} \frac{m}{1+m}\right] \exp \left[-\frac{1+\frac{\lambda_{z}}{1+m}}{\lambda_{\Delta}^{0}}\right]
$$

и $\lambda_{z}$ и $\lambda_{\Delta}^{0}$ зависят от $m($ см. (55)). Уравнение (69) по виду совпадает с соответствующим уравнением Абрикосова и Горькова [8]. Однако величина $T_{C 0}$ и параметр рассеяния $\rho$ в нашем уравнении переопределены и сушественно зависят от параметра неадиабатичности $m$ и импульса обрезания $Q_{c}$. На основании $(69)$ имеем

$$
\begin{aligned}
& T_{C}=T_{C 0}-\frac{\pi^{2}}{2} \frac{\Gamma}{2 \pi} f_{c}(m) \quad \text { при } \rho \ll 1, \\
& T_{C}^{2}=6 \frac{\Gamma^{2}}{\pi^{2}}\left[f_{c}(m)\right]^{2} \ln \frac{\pi T_{C 0}}{\gamma_{\ni} \Gamma f_{c}(m)} \quad \text { при } \rho \gg 1 .
\end{aligned}
$$


Отсюда для критической концентрации примеси, когда $T_{C}=0$, получаем

$$
\Gamma_{\mathrm{cr}}=\frac{\pi T_{C 0}}{2 \gamma_{\ni} f_{c}(m)} .
$$

Эти формулы отличаются от соответствующих выражений из работы [8] определением $T_{C 0}(m)(70)$ и наличием функции $f_{c}(m)$. Таким образом, примесная зависимость величины $T_{C}$ и значение $\Gamma_{\text {cr }}$ зависят от параметра Мигдала $m$, а следовательно, от свойств основного вешества, в которое вводится магнитная примесь. Интересно, что за счет эффекта неадиабатичности происходит перенормировка не только величины $T_{C 0}$, но и параметра рассеяния электронов на магнитной примеси $\rho$, которое уменьшается $\left(f_{c}(m)<1\right)$. Такой результат можно объяснить ослаблением распаривающего влияния парамагнитной примеси из-за дополнительного электрон-фононного взаимодействия, возникаюшего из диаграмм с пересечением примесных и фононных линий. Это приведет, в свою очередь, к более медленному убыванию величины $T_{C}$ с ростом концентрации примеси по сравнению со случаем обычных сверхпроводников $(m=0)$. Слабое убывание $T_{C}$ с ростом концентрации примеси обнаружено в иттриевых керамиках при замешении меди в плоскости $\mathrm{CuO}_{2}$ парамагнитными примесями типа $\mathrm{Ni}[11,12]$.

На основании (71) для изотопического коэффициента

$$
\alpha=-\frac{d \ln T_{C}}{d \ln M} \quad \text { при } \quad \rho \ll 1
$$

получаем

$$
\alpha=\frac{\alpha_{0}-\frac{\pi \Gamma}{8 T_{C}} \frac{d f_{c}(m)}{d \ln m}}{1-\frac{\pi \Gamma}{4 T_{C}} f_{c}(m)}
$$

где

$$
\alpha_{0}=-\frac{d \ln T_{C 0}}{d \ln M}
$$

a $f_{c}(m)$ приближенно записьвается в виде

$$
\begin{aligned}
f_{c}(m) & \approx 1-\lambda \frac{2 m+1}{m+1}, \\
\frac{d f_{c}(m)}{d \ln m} & \approx-\lambda \frac{2 m^{2}-1}{(m+1)^{2}} .
\end{aligned}
$$

Отсюда следует, что $f_{c}(m)<1$, а

$$
\frac{d f_{c}(m)}{d \ln m}\left\{\begin{array}{l}
>0 \text { при } 0<m<\frac{1}{\sqrt{2}}, \\
<0 \text { при } \frac{1}{\sqrt{2}}<m .
\end{array}\right.
$$

Следовательно, примесная зависимость изотопического коэффишиента определяется параметром Мигдала $m$.

При $m=0$ мы имеем рост коэффициента $\alpha$ с ростом концентрации примеси, и это соответствует случаю обычных сверхпроводников [11]. Если $0<m<1 / \sqrt{2}$, то с ростом концентрации примеси происходит убывание как числителя, так и знаменателя выражения (73) и результат будет определяться значением параметра $m$. При $1 / \sqrt{2}<m$ происходит рост величины $\alpha$ за счет роста числителя и убыли знаменателя выражения (73) с ростом концентрации примеси. 


\section{5. ЗАКЛЮЧЕНИЕ}

В данной работе была поставлена задача определения температуры сверхпроводящего перехода и изотопического эффекта в неадиабатических сверхпроводниках (в которых энергия Ферми и энергия Дебая одного порядка) при наличии в системе хаотически распределенной магнитной примеси. Мы исходили из гамильтониана (1), в котором учитывали электрон-фононное и примесное взаимодействия. Применяя теорию возмушений по электрон-фононному и примесному взаимодействиям, усреднение по хаотически распределенным положениям примесных атомов и ориентациям их спинов, мы вывели уравнения для функций Грина (нормальной и аномальных) и выписали выражения для их массовых операторов, которые включают также и вершинные поправки электрон-фононного взаимодействия, а также вклады от диаграмм, содержаших пересечение электрон-фононных и электрон-примесных линий. Выполнив усреднение по поверхности Ферми, мы получили для массовых операторов формулы (24) и (25), отсюда следует самосогласованная система уравнений (26)-(28). Учет указанных выше дополнительных диаграмм приводит к перенормировке константы электрон-фононного взаимодействия, а также к перенормировке параметра электрон-примесного рассеяния (52)-(54). Для получения наглядных результатов мы перешли к случаю слабой электрон-фононной связи $\left(\omega_{0} / T_{C} \ll 1\right)$, а также выполнили ряд других упрошений, как это делается в обычной неадиабатической теории. Мы показали, что примесное взаимодействие слабо перенормирует константу электрон-фононного взаимодействия. Учет же неадиабатичности приводит к сушественному переопределению величины $T_{C 0}$ и параметра $\rho$ рассеяния электронов на магнитной примеси (67). Так как $f(m)<1$, мы имеем в уравнении (71) более слабое убывание величины $T_{C}$ c ростом концентрации примеси при малых концентрациях, чем в случае обычных сверхпроводников. Определено также, что критические концентрации примеси (72), при которых $T_{C}$ обрашается в нуль, значительно больше, чем в случае обычных сверхпроводников, за счет больших значений $T_{C 0}$ и $f(m)<1$

Таким образом, можно сказать, что теория Абрикосова и Горькова [8] работает также и в случае неадиабатических систем с той разницей, что $T_{C 0}$ и $\rho$ переопределены и существенно зависят от параметра неадиабатичности $m$. Зависимость же $T_{C}$ от концентрации примеси, которую можно определить экспериментально, приводит к существенным количественным различиям для различных сверхпроводников. Это связано с наличием параметра неадиабатичности $m$ и импульса обрезания $q_{c}$, которые определяют свойства основного вешества. Сушественным также оказывается наличие параметра Мигдала $m$ и величины $q_{c}$ в примесной зависимости изотопического коэффициента $\alpha$.

Как уже отмечалось, мы исходили из простой модели - гамильтониана Фрелиха, учитывая наличие парамагнитной примеси в системе. Наряду со специфическими эффектами зонной структуры [13-16] во всех ВТСП-материалах сушественную роль играют сильные электронные корреляции, индуцируемые кулоновским взаимодействием [17-19]. Многие свойства этих материалов могут быть поняты на основании модели типа хаббардовской. Однако возможен и другой подход $[3,5]$, которого мы и придержи- 
ваемся в данной работе, а именно, включающий косвенный учет сильных электронных корреляций через их влияние на электрон-фононное взаимодействие. Такое рассмотрение основывается на результатах исследований [20], согласно которым из-за сильных электронных корреляций константа электрон-фононного взаимодействия $g(q)$ с обрезанием по импульсу $q_{c}$ принимает вид, использованный в модельном представлении (29). В системах с сильными электронными корреляциями (малыми $q_{c}$ ) рассмотренные выше вершинные функции $P_{V}$ и $P_{c}$ являются положительными и, следовательно, благоприятными для появления высоких значений $T_{C}$ (подробнее см. $[3,5]$ ).

При решении поставленной задачи мы учитывали, что в системе нарушается теорема Мигдала ( $\left.\omega_{\mathrm{D}} \sim E_{\mathrm{F}}\right)$, а также имеются сильные электронные корреляции (обрезание электрон-фононного и электрон-примесного взаимодействий по малым $q_{c}$ ). Эти свойства присуши ВТСП-материалам. Следовательно, можно полагать, что предложенная теория улавливает качественное поведение величин $T_{C}$ и $\alpha$ как функций от концентрации примеси.

В любом случае эта модель ближе к ВТСП-материалам, чем теория АбрикосоваГорькова [8], применимая к обычным сверхпроводникам. В частности, при $\omega_{\mathrm{D}}=E_{\mathrm{F}}$ на основании формул для коэффициента изотопического эффекта получаем для случая чистого сверхпроводника $\alpha \approx 0.2$ и рост этого коэффициента от 0.2 до 1.5 с ростом концентрации примеси, что наблюдается в лантановых керамиках $[11,21]$.

Отметим, что другой важной особенностью ВТСП-материалов является перекрытие энергетических полос на поверхности Ферми. Учет этой особенности приводит к возникновению дополнительной примесной зависимости величин $T_{C}$ и $\alpha$ из-за канала межзонного рассеяния на примесном потенциале [22].

\section{ПРИЛОЖЕНИЕ}

При вычислении дополнительных примесных вкладов, возникаюших при учете пересечений примесных и электрон-фононных линий, используем нулевые функции Грина по взаимодействию.

Воспользуемся представлением

$$
\begin{aligned}
\Gamma_{V}\left(\vec{p} \vec{p}_{1}, \Omega \Omega_{1}\right)= & \left(\frac{2 k_{\mathrm{F}}}{q_{c 1}}\right)^{2} \frac{W_{+}}{N_{0} V} \sum_{\vec{p}_{2}} \theta\left(q_{c 1}-\left|\vec{p}-\vec{p}_{2}\right|\right) \frac{1}{\left(i \Omega-\varepsilon_{\vec{p}_{2}}\right)\left(i \Omega_{1}-\varepsilon_{\left.\vec{p}_{1}+\vec{p}_{2}-\vec{p}\right)}\right.}= \\
= & W_{+}\left(\frac{2 k_{\mathrm{F}}}{q_{c 1}}\right)^{2} \int_{-\pi}^{\pi} \frac{d \varphi}{2 \pi} \int_{0}^{\pi} \frac{\sin \alpha d \alpha}{2} \int_{-E / 2}^{E / 2} d \varepsilon_{\vec{p}_{2}} \theta\left(q_{c 1}-\left|\vec{p}-\vec{p}_{2}\right|\right) \times \\
& \times \frac{1}{\varepsilon_{\vec{p}_{1}+\vec{p}_{2}-\vec{p}}-\varepsilon_{\vec{p}_{2}}+i\left(\Omega-\Omega_{1}\right)} \times \\
& \times\left[\frac{1}{\varepsilon_{\vec{p}_{2}}-i \Omega}-\frac{1}{\varepsilon_{\vec{p}_{1}+\vec{p}_{2}-\vec{p}}-i \Omega_{1}}\right] .
\end{aligned}
$$


Выберем линейный закон дисперсии $\left(p \sim p_{1} \sim p_{2} \sim k_{\mathrm{F}}\right)$ :

$$
\begin{aligned}
\varepsilon_{\vec{p}_{1}+\vec{p}_{2}-\vec{p}} & =v_{\mathrm{F}}\left|\vec{p}_{1}+\vec{p}_{2}-\vec{p}\right|-\mu= \\
& =v_{\mathrm{F}} \sqrt{p_{1}^{2}+p_{2}^{2}+p^{2}-2 p p_{2} \cos \alpha-2 p p_{1} \cos \theta+2 p_{1} p_{2} \cos \beta}-\mu .
\end{aligned}
$$

При малых значениях $q_{c}$ и $Q_{c 1}$ это выражение можно представить в виде [5]

$$
\varepsilon_{\vec{p}_{1}+\vec{p}_{2}-\vec{p}}=\varepsilon_{\vec{p}_{2}}+E Q^{2}(1-\cos \alpha)+E Q \sqrt{1-Q^{2}} \sin \alpha \cos \varphi \approx \varepsilon_{\vec{p}_{2}}+E Q \alpha \cos \varphi
$$

Здесь

$$
Q=\frac{q}{2 k_{\mathrm{F}}}
$$

и $q=\left|\vec{p}-\vec{p}_{1}\right|-$ передаваемый импульс.

Для малых $q_{c 1}$ заменяем

$$
\theta\left(q_{c 1}-\left|\vec{p}-\vec{p}_{2}\right|\right) \cong \theta\left(\cos \alpha+2 Q_{c 1}^{2}-1\right) \approx \theta\left(2 Q_{c 1}-\alpha\right)
$$

Тогда (П.1) с учетом (П.2) и (П.3) приводится к виду

$$
\begin{aligned}
\Gamma_{V}\left(\vec{p} \vec{p}_{1}, \Omega \Omega_{1}\right)= & W_{+}\left(\frac{2 k_{\mathrm{F}}}{q_{c 1}}\right)^{2} \int_{-\pi}^{\pi} \frac{d \varphi}{2 \pi} \int_{0}^{2 Q_{c 1}} \frac{\alpha d \alpha}{2} \frac{1}{E Q \alpha \cos \varphi+i\left(\Omega-\Omega_{1}\right)} \times \\
& \times\left[\int_{-E / 2}^{E / 2} \frac{d \varepsilon_{\vec{p}_{2}}}{\varepsilon_{\vec{p}_{2}}-i \Omega}-\int_{-E / 2}^{E / 2} \frac{d \varepsilon_{\vec{p}_{2}}}{\varepsilon_{\vec{p}_{2}}+E Q \alpha \cos \varphi-i \Omega_{1}}\right]= \\
= & W_{+}\left(\frac{2 k_{\mathrm{F}}}{q_{c 1}}\right)^{2} \int_{-\pi}^{\pi} \frac{d \varphi}{2 \pi} \int_{0}^{2 Q_{c 1}} \frac{\alpha d \alpha}{2} \times \\
& \times \frac{K\left(\Omega \Omega_{1}\right)+L\left(\Omega \Omega_{1}\right)(E Q \alpha \cos \varphi)^{2}}{(E Q \alpha \cos \varphi)^{2}+\left(\Omega-\Omega_{1}\right)^{2}},
\end{aligned}
$$

где

$$
\begin{aligned}
& K\left(\Omega \Omega_{1}\right)=2\left(\Omega-\Omega_{1}\right)\left[\operatorname{arctg} \frac{E}{2 \Omega}-\operatorname{arctg} \frac{E}{2 \Omega_{1}}\right], \\
& L\left(\Omega \Omega_{1}\right)=\frac{E\left(\Omega-\Omega_{1}\right)}{\Omega_{1}^{3}\left(1+\frac{E^{2}}{4 \Omega_{1}^{4}}\right)^{2}}-\frac{E}{\frac{E^{2}}{4}+\Omega_{1}^{2}} .
\end{aligned}
$$

Выполнив интегрирования в (П.4), получаем

$$
\begin{aligned}
\Gamma_{V}\left(\vec{p} \vec{p}_{1}, \Omega \Omega_{1}\right)= & W_{+}\left\{L\left(\Omega \Omega_{1}\right)+\frac{1}{2 E^{2} Q^{2} Q_{c 1}^{2}}\left[K\left(\Omega \Omega_{1}\right)-\left(\Omega-\Omega_{1}\right)^{2} L\left(\Omega \Omega_{1}\right)\right] \times\right. \\
& \left.\times\left[\sqrt{1+\left(\frac{2 E Q Q_{c 1}}{\Omega-\Omega_{1}}\right)^{2}}-1\right]\right\} .
\end{aligned}
$$


Выражение для $\Gamma_{c}^{\mathrm{I}}$ представим в виде

$$
\begin{aligned}
\Gamma_{c}^{\mathrm{I}}\left(\vec{p} \vec{p}_{1}, \Omega \Omega_{1}\right)= & W_{-}\left(\frac{2 k_{\mathrm{F}}}{q_{c}}\right)^{2} \int_{-\pi}^{\pi} \frac{d \varphi}{2 \pi} \int_{0}^{\pi} \frac{\sin \alpha d \alpha}{2} \int_{-E / 2}^{E / 2} d \varepsilon_{\vec{p}_{2}} \theta\left(q_{c}-\left|\vec{p}-\vec{p}_{2}\right|\right) \times \\
& \times \frac{1}{\varepsilon_{\vec{p}_{2}}-\varepsilon_{\vec{p}_{2}-\vec{p}_{1}-\vec{p}}-i\left(\Omega-\Omega_{1}\right)} \times \\
& \times\left[\frac{1}{\varepsilon_{\vec{p}_{2}-\vec{p}_{1}-\vec{p}}+i \Omega_{1}}-\frac{1}{\varepsilon_{\vec{p}_{2}}-i \Omega}\right] .
\end{aligned}
$$

Здесь [5]

$$
\begin{aligned}
\varepsilon_{\vec{p}_{2}-\vec{p}_{1}-\vec{p}} & =v_{\mathrm{F}}\left|\vec{p}_{2}-\vec{p}_{1}-\vec{p}\right|-\mu= \\
& =v_{\mathrm{F}} \sqrt{p_{2}^{2}+p_{1}^{2}+p^{2}-2 p_{2} p_{1} \cos \beta-2 p_{2} p \cos \alpha+2 p_{1} p \cos \theta}-\mu \approx \\
& \approx \varepsilon_{\vec{p}_{2}}+\eta, \quad \eta=E\left(1-Q^{2}\right) \frac{\alpha^{2}}{2}-E Q \sqrt{1-Q^{2}} \alpha \cos \beta .
\end{aligned}
$$

В результате после интегрирования по энергии получаем

$$
\Gamma_{c}^{\mathrm{I}}\left(\vec{p} \vec{p}_{1}, \Omega \Omega_{1}\right)=W_{-}\left(\frac{2 k_{\mathrm{F}}}{q_{c}}\right)^{2} \int_{-\pi}^{\pi} \frac{d \varphi}{2 \pi} \int_{0}^{2 Q_{c}} \frac{\alpha d \alpha}{2} \frac{K\left(-\Omega_{1} \Omega\right)+\eta^{2} L\left(-\Omega_{1} \Omega\right)}{\eta^{2}+\left(\Omega+\Omega_{1}\right)^{2}}
$$

Выполнив в этом выражении интегрирование подобно тому, как это было сделано в [5], получаем

$$
\begin{aligned}
\Gamma_{c}^{\mathrm{I}}\left(\vec{p} \vec{p}_{1}, \Omega \Omega_{1}\right)= & W_{-}\left\{L\left(-\Omega_{1} \Omega\right)+\frac{1}{2 E Q_{c}^{2}\left(1-Q^{2}\right)}\left[\frac{K\left(-\Omega_{1} \Omega\right)}{\Omega+\Omega_{1}}-\left(\Omega+\Omega_{1}\right) L\left(-\Omega_{1} \Omega\right)\right] \times\right. \\
& \left.\times \operatorname{arctg} \frac{2 E Q_{c}^{2}\left(1-Q^{2}\right)}{\Omega+\Omega_{1}}\right\} .
\end{aligned}
$$

Нетрудно видеть, что если в приведенных выше формулах положить $\Omega=0$, а $\Omega_{1}=\omega_{0}$, то получим $\Gamma_{V} \sim W_{+} / \omega_{0} \ll 1$ и $\Gamma_{c} \sim W_{-} / \omega_{0} \ll 1$. 


\section{Список литературы}

[1] G. J. Uemura et al. Phys. Rev. Lett. 1991. V. 66. P. 2665.

[2] V.Z. Kresin, S. A. Wolf. Phys. Rev. B. 1987. V. 41. P. 4278.

[3] L. Pietronero, S. Strässler, C. Grimaldi. Phys. Rev. B. 1995. V. 52. P. 10516.

[4] А.Б. Мигдал. ЖЭТФ. 1958. Т. 34. С. 1438.

[5] C. Grimaldi, L. Pietronero, S. Strässler. Phys. Rev. B. 1995. V. 52. P. 10530.

[6] A. С. Элиашберг. ЖЭЭФ. 1960. Т. 38. С. 966.

[7] P. W. Anderson. J. Phys. Chem. Solids. 1959. V. 11. P. 26.

[8] А.А. Абрикосов, Л. П. Горьков. ЖЭТФ. 1960. Т. 39. С. 1781.

[9] А.А. Абрикосов, Л. Д. Горьков, И.Е. Дзялошинский. Методы квантовой теории поля в статистической физике. М.: Наука, 1962.

[10] W. L. McMillan. Phys. Rev. 1968. V. 167. P. 331.

[11] Н. Н. Плакида. Высокотемпературные сверхпроводники. М.: Междунар. прогр. образования, 1996.

[12] J. M. Taracson et al. Phys. Rev. B. 1988. V. 37. P. 7458.

[13] В. А, Москаленко, М.Е. Палистрант, В. М. Вакалюк. УФН. 1991. Т. 161. С. 155.

[14] Ф.Г. Кочорбә, М. Е. Палистрант. ЖЭЭТФ. 1993. Т. 104. С. 3084; ТМФ. 1993. Т. 96. C. 459 .

[15] H. Krakauer, W. E. Pickett. Phys. Rev. Lett. 1988. V. 60. P. 1665.

[16] J.F. Herman, R. V. Kasowski, W. J. Hsau. Phys. Rev. B. 1987. V. 36. P. 6904.

[17] P. W. Anderson, J. R. Schrieffer. Phys. Today. 1991. V. 20. P. 84.

[18] P. Fulde. Physica C. 1988. V. 153-155. P. 1769.

[19] R. W. Lof. Phys. Rev. Lett. 1992. V. 68. P. 3924.

[20] M. L. Kulic, R. Zeyher. Phys. Rev. B. 1984. V. 49. P. 4395; Physica B. 1994. V. 199-200. P. 358.

[21] N. Babushkina, A. Inushkin, V. Ozhogin et al. Physica C. 1991. V. 185-189. P. 901; 1994. V. 235-240. P. 845.

[22] В. А. Москаленко, М. Е. Палистрант. ЖЭТФ. 1965. Т. 49. С. 770; ДАН СССР. 1965. T. 162. C. 539. 